\title{
An Integrated Framework for MC-CDMA Reception in the Presence of Frequency Offsets, Phase Noise, and Fast Fading
}

\author{
Tamer A. Kadous, Member, IEEE, and Akbar M. Sayeed, Senior Member, IEEE
}

\begin{abstract}
The combination of code-division multiple-access and multicarrier (MC) modulation has been proposed to develop high data-rate wireless communication systems. Due to the longer symbol duration in comparison with single-carrier systems, MC systems are more sensitive to various imperfections, including phase noise and frequency offsets due to local oscillators and Doppler spreading due to motion that results in temporal channel variations. The performance of current systems is significantly limited by these imperfections because they disperse the transmitted power in a particular subcarrier into adjacent subcarriers, thereby causing interference between the subcarriers at the receiver. We consider a single-user communication system and use a canonical model for the received signal that efficiently captures the effects of all impairments. The model uses Fourier basis functions that are fixed for all imperfections while the expansion coefficients depend on imperfections. Using the model, we introduce a receiver structure that implements the matched filter (MF), and hence, optimal. The MF is implemented through a Rake receiver in the frequency domain. The receiver fully compensates for frequency offsets as well as phase noise, thereby eliminating the performance loss due to these factors. Furthermore, in contrast to existing designs, it delivers improved performance under fast fading by exploiting Doppler diversity. Finally, the same integrated receiver structure works for all imperfections eliminating the need for devising a separate correction technique for each.
\end{abstract}

Index Terms-Code-division multiple-access (CDMA), diversity, Doppler shifts, fast fading, frequency offset, multicarrier (MC), orthogonal frequency-division multiplexing (OFDM), phase noise.

\section{INTRODUCTION}

$\mathbf{E}$ MERGING wireless personal communication services require high bandwidth to support multimedia formats. Direct-sequence code-division multiple-access (DS-CDMA) has emerged as a core wireless technology in the third generation and emerging standards. However, conventional CDMA systems are fundamentally limited in their ability to deliver high data rates due to implementational issues associated

Manuscript received July 12, 2002; revised February 3, 2003; accepted March 21,2003 . The editor coordinating the review of this paper and approving it for publication is A. Swami. This work was supported in part by the Wisconsin Alumni Research Foundation and in part by the National Science Foundation under the CAREER Grant CCR-9875805.

T. A. Kadous was with the Department of Electrical and Computer Engineering, University of Wisconsin-Madison, Madison, WI 53706 USA. He is now with Qualcomm, Inc., San Diego, CA 92121 USA (e-mail: kadous@ qualcomm.com).

A. M Sayeed is with the Department of Electrical and Computer Engineering, University of Wisconsin-Madison, Madison, WI 53706 USA (e-mail: akbar@engr.wisc.edu).

Digital Object Identifier 10.1109/TWC.2004.830828 with higher chip rates and complexity issues related to higher intersymbol interference (ISI). These impairments put severe constrains on the data rate supportable by single-carrier (SC)-CDMA systems [1].

Multicarrier (MC) modulation, often called orthogonal frequency-division multiplexing (OFDM), has drawn a lot of attention due to its ability to support high rates while ameliorating ISI and fading. In OFDM, the high rate stream is split into a number of parallel lower rate ones that are transmitted over narrowband orthogonal subcarriers. The longer symbol duration of lower rate streams, in conjunction with a guard time (cyclic prefix), significantly reduces the effects of ISI. An attractive aspect of OFDM is that modulation and demodulation can be implemented efficiently by a discrete Fourier transform (DFT).

MC-CDMA, a combination of CDMA and OFDM, has been proposed to support high data rates in CDMA systems (see, for example, [1] and the references therein). Basically, three main designs exist in literature namely, MC-CDMA [2], MC-DS-CDMA [3], and multitone (MT)-CDMA [4]. In MC-CDMA, the signature code is applied across a number of orthogonal subcarriers in the frequency domain. In MC-DS-CDMA, the data stream is first divided into a number of substreams. Each substream is spread in time through a signature code and then transmitted over one of a set of orthogonal subcarriers. The MT-CDMA system undergoes similar operations as MC-DS-CDMA except that the different subcarriers are not orthogonal after spreading. This allows higher spectral efficiencies and longer spreading codes, however, different substreams interfere with one another.

This paper is in the context of MC-CDMA. In an MC-CDMA system, the lower data rate supported by each subcarrier is manifested in longer symbol and chip durations. In a properly designed system, each subchannel encounters flat fading, thereby eliminating the need for channel equalization. Furthermore, the system exploits frequency selectivity for diversity via the different subcarriers. MC-CDMA also requires a lower-speed parallel-type processing compared to fast serial-type processing in a SC-CDMA system. These features make MC-CDMA a strong candidate for high-rate wireless communications.

Though MC-CDMA is robust to ISI, its performance and ease of implementation critically depend on the orthogonality between subcarriers, a property that is destroyed by nonideal system characteristics encountered in practice. These nonidealities include frequency offsets and phase noise due to local oscillators at the transmitter and/or receiver, as well as Doppler 
effects due to fast fading. MC-CDMA systems are more sensitive to these imperfections than SC-CDMA systems due to longer symbol durations. For example, the channel may appear almost constant over one (short) symbol duration in an SC-CDMA system while it may exhibit faster variation over the longer MC-CDMA symbol. The loss of orthogonality between subcarriers is due to the dispersion of signal power from a particular subcarrier into adjacent frequencies which results in intercarrier interference (ICI). Since existing MC-CDMA receivers process each subchannel separately, they collect only part of the transmitted power in each subcarrier in addition to suffering from ICI. These imperfections severely limit the performance of existing MC-CDMA systems [5]-[10].

This paper addresses the design and analysis of MC-CDMA systems in the presence of frequency offsets, phase noise, and fast fading. We consider a single-user system to focus on the effects of imperfections. Multiuser systems are discussed in [11]. We assume a sufficiently large number of carriers so that ISI is negligible in each subcarrier. We introduce an integrated receiver structure for combating the above-mentioned imperfections. The receiver is based on a canonical model for the received signal that efficiently captures the effects of the imperfections. In essence, each subcarrier is decoded by jointly processing it and a subset of adjacent subcarriers. Our results demonstrate that, with slightly higher complexity, performance degradation due to imperfections can be virtually eliminated. Furthermore, in contrast to existing designs, the proposed receiver exploits Doppler diversity [12] to deliver improved performance under fast fading.

Section II reviews the channel model and receiver structure in conventional MC-CDMA systems. Section III develops the proposed receiver structure in the presence of imperfections and analyze performance. Section IV discusses the problem of channel estimation in the context of the proposed receiver structure. Sections V-VII assess the performance of the proposed receiver in the presence of frequency offsets, fast fading, and phase noise, respectively. Section VIII presents numerical results depicting a variety of scenarios. Section IX presents some concluding remarks.

\section{SYSTEM MODEL}

In this section, we review a typical MC-CDMA system. We first describe the channel model. A typical MC-CDMA transmitter and receiver then follow.

\section{A. Channel Model}

We review some relevant statistical characteristics of the widely used wide sense stationary uncorrelated scattering (WSSUS) channel model [13], [14] that is adopted in this paper. The WSSUS model captures temporal channel variations and is characterized by a randomly time-varying impulse response $h(t, \tau)$. For fixed $\tau, h(t, \tau)$ is modeled as a complex WSS Gaussian process in $t$. The channel response for different values of $\tau$ is uncorrelated. The time varying transfer function of the channel is

$$
\tilde{h}(t, f)=F T_{\{\tau\}}\{h(t, \tau)\}=\int_{-\infty}^{\infty} h(t, \tau) e^{-j 2 \pi f \tau} d \tau
$$

where $F T_{\{t\}}$ denotes the Fourier transform with respect to the variable $t$. Under the WSSUS assumption, $\tilde{h}(t, f)$ is a WSS process in both $t$ and $f$. The statistics $\tilde{h}(t, f)$ are characterized by the spaced-time, spaced-frequency correlation function $\phi(\Delta t, \Delta f)=E\left[\tilde{h}(t, f) \tilde{h}^{*}(t-\Delta t, f-\Delta f)\right]$. Under the assumption that all multipaths have the same spaced-time correlation function, $\phi_{\Delta t}(\Delta t)=E\left[\tilde{h}(t,.) \tilde{h}^{*}(t-\Delta t,).\right], \phi(\Delta t, \Delta f)$ becomes separable [15]

$$
\phi(\Delta t, \Delta f)=\phi_{\Delta t}(\Delta t) \psi_{\Delta f}(\Delta f)
$$

where $\psi_{\Delta f}(\Delta f)=E\left[\tilde{h}(., f) \tilde{h}^{*}(., f-\Delta f)\right]$ is the spaced-frequency correlation function. This assumption is used in the performance analysis in Section VIII.

The duration over which the channel is strongly correlated is called the coherence time, $\Delta t_{c}$. Define the Doppler power spectrum function to be $\Phi_{\theta}(\theta)=F T_{\{\Delta t\}}\left\{\phi_{\Delta t}(\Delta t)\right\}$. The support of $\Phi_{\theta}(\theta)$ is called the Doppler spread $B_{d}$ of the channel; $\Phi_{\theta}(\theta)=0,|\theta|>B_{d}$. The coherence time and Doppler spread are inversely related: $\Delta t_{c} \approx 1 / 2 B_{d}$.

The channel power at different values of $\tau$ is called the multipath intensity profile: $\Psi_{\tau}(\tau)=E\left[|h(., \tau)|^{2}\right]$. The support of $\Psi_{\tau}(\tau)$ is called the multipath spread $T_{m}$ of the channel; $\Psi_{\tau}(\tau)=0$ for $\tau<0$ or $\tau>T_{m}$. The Fourier transform of $\Psi_{\tau}(\tau)$ is the spaced-frequency correlation function $\psi_{\Delta f}(\Delta f)=F T_{\{\tau\}}\left\{\Psi_{\tau}(\tau)\right\}$. The frequency span over which the channel is strongly correlated is called the coherence bandwidth, $\Delta f_{c}$. Coherence bandwidth and multipath spread are inversely related: $\Delta f_{c} \approx 1 / T_{m}$

Throughout the paper, we denote the duration of the signaling waveform with $T$ and its essential two-sided bandwidth with $B$. We consider a frequency selective $\left(B>\Delta f_{c}\right)$ channel under both slow $\left(T \ll \Delta t_{c}\right.$ ) and fast ( $T \geq \Delta t_{c}$ ) fading. We note that the effect of fast fading becomes significant for relatively small $(\approx 0.2)$ values of $T / \Delta t_{c} \approx 2 B_{d} T[12]$.

\section{B. MC-CDMA Transmitter}

In DS-CDMA, the transmitted spread spectrum signal occupies the entire bandwidth $B$ and duration $T$. The idea behind MC-CDMA is to transmit the data on parallel channels, each parallel channel occupying a fraction of the bandwidth of the original CDMA signal. In a typical MC-CDMA system, shown in Fig. 1, the signature code $\left\{a_{n}, n=1,2, \ldots, N\right\}$ of a particular user is spread among a set of $N$ orthogonal subcarriers each carrying the same information bit $b$. Thus, each chip modulates one of $N$ orthogonal subcarriers. This process is done by the $N$-point DFT block. A guard time is then added to reduce the effects of ISI. The subcarriers are separated by $1 / T$ and the two-sided null-to-null bandwidth associated with each subcarrier is $B_{o}=2 / T$. The objective is to make $B_{o}$ smaller than $\Delta f_{c}$ so that each subcarrier encounters flat fading.

Due to negligible ISI, we focus on one shot detection and, without loss of generality, study the signal model for the first symbol of User 1 . The transmitted signal can be written as

$$
s(t)=b \sum_{n=1}^{N} a_{n} q(t) e^{j 2 \pi f_{n} t}, \quad 0 \leq t \leq T
$$



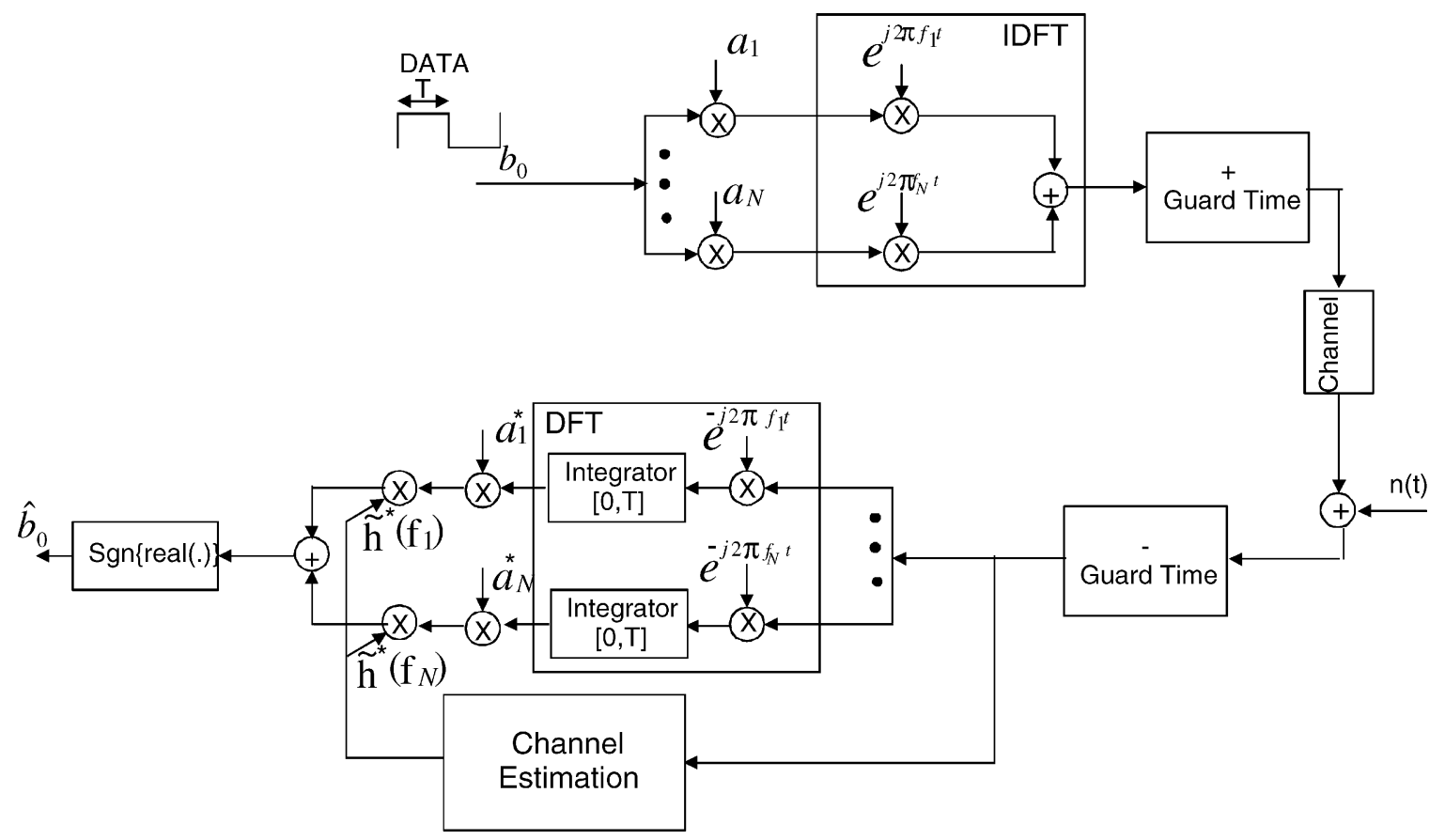

Fig. 1. Typical MC-CDMA system.

where $q(t)=1 / \sqrt{T}, 0 \leq t \leq T$ is the normalized symbol waveform, $b$ is the transmitted bit, and $f_{n}=n / T$ is the $n$th subcarrier frequency. As mentioned earlier, we consider a single-user system to focus on the effects of imperfections.

\section{Conventional MC-CDMA Receiver}

Consider a conventional MC-CDMA system with no imperfections in a slowly fading channel. At the receiver, the guard time (cyclic prefix) is discarded and the signal is fed to an $N$-point DFT block to separate the subcarriers. The received signal for the first symbol can be written as

$$
r(t)=b \sum_{n=1}^{N} a_{n} \tilde{h}\left(f_{n}\right) q(t) e^{j 2 \pi f_{n} t}+n(t), \quad 0 \leq t \leq T
$$

where $\tilde{h}\left(f_{n}\right)$ is the channel coefficient of the $n$th subcarrier and $n(t)$ is complex additive white Gaussian noise with power spectral density $\sigma^{2}$. The test static $z_{n}$ resulting from projecting $r(t)$ onto the $n$th subcarrier is

$$
z_{n}=\frac{1}{\sqrt{T}} \int_{0}^{T} r(t) e^{-j 2 \pi f_{n} t} d t=b a_{n} \tilde{h}\left(f_{n}\right)+v_{n}
$$

where $\left\{v_{n}\right\}$ are independent zero-mean Gaussian random variables with variance $\sigma^{2}$. With perfect channel estimates, the bit decision is given by

$$
\hat{b}=\operatorname{sign}\left[\operatorname{real}\left\{\sum_{n=1}^{N} a_{n}^{*} \tilde{h}^{*}\left(f_{n}\right) z_{n}\right\}\right] .
$$

We note that this receiver provides $L$-fold diversity, where $L=$ $\left\lceil T_{m} B\right\rceil$ is the number of resolvable multipaths as well as the number of uncorrelated coherence bands within the available bandwidth, i.e., $B / \Delta f_{c}=B T_{m}$ [13].

\section{PROPOSED FRAMEWORK}

In the presence of fast fading, Doppler shifts, frequency offset, and/or phase noise, the orthogonality between subcarriers is destroyed. The matched filter (MF) output (5) will suffer from leakage from the other active subcarriers [5]-[7], [16]. This leakage disperses the energy of a particular subcarrier over the adjacent subcarriers. Thus, it is necessary to jointly process the test statistics from different subcarriers. We introduce a universal receiver structure that combats all these impairments in an integrated fashion. It fully eliminates the performance loss due to frequency offsets and phase noise. Moreover, in contrast to existing receivers, it exploits temporal channel variations for improved performance via Doppler diversity [12].

\section{A. Integrated Receiver Structure}

The information-bearing signal in (4) in the ideal channel is a linear combination of the fixed basis functions $u_{n}(t)=$ $q(t) e^{j 2 \pi f_{n} t}, n=1,2, \ldots N$, with $\tilde{h}\left(f_{n}\right)$ as the corresponding expansion coefficients. In the presence of imperfections, the received signal corresponding to the first symbol can be represented as

$$
r(t)=b \sum_{n=1}^{N} a_{n} w\left(t, f_{n}\right) q(t) e^{j 2 \pi f_{n} t}+n(t), \quad 0 \leq t \leq T
$$

$$
w(t, f)=\tilde{h}(t, f) e^{j 2 \pi f_{\text {off }} t} e^{j \varrho(t)}
$$

where $\tilde{h}\left(t, f_{n}\right)$ accounts for the time-varying frequency selective channel, $e^{j 2 \pi f_{\text {off }} t}$ accounts for frequency offset between the transmitter and receiver oscillators, and $e^{j \varrho(t)}$ accounts for phase noise. ${ }^{1}$ While the net effect of these imperfections is to

${ }^{1}$ This model corresponds to phase noise at the transmitter. Our development can be easily extended to the case of having the phase noise at the receiver or at both the transmitter and the receiver. 


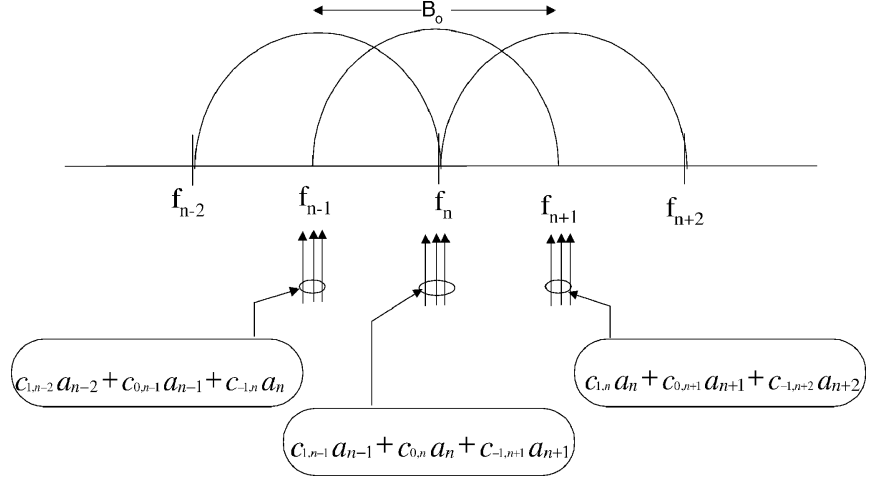

Fig. 2. Coefficients associated with the $(n-1)$ th, $n$ th, and $(n+1)$ th subcarriers at the receiver for $K_{l}=K_{u}=1$.

cause spectral dispersion, their nature is different. In particular, $\tilde{h}\left(t, f_{n}\right)$ and $e^{j \varrho(t)}$ can result in a continuous spectral spread around each subcarrier. On the other hand, $e^{j 2 \pi f_{\text {off }} t}$ causes a frequency shift relative to the exact subcarrier frequency. The net result is that compared to the ideal system (4), the channel coefficients in (7) are no longer constant over a symbol duration. Temporal variations in $w\left(t, f_{n}\right)$ are manifested at the receiver as spectral dispersion - the transmitted energy in each subcarrier is dispersed in a band of frequencies around the subcarrier frequency.

The key idea of this paper is that, due to the finite symbol duration, any arbitrary spectral spreading around each subcarrier can be represented in terms of a finite number of discrete frequencies via a Fourier series [12], [14], [17]. More specifically, let $\tilde{w}\left(t, f_{n}\right)=w\left(t, f_{n}\right) I_{[0, T]}(t)$ denote the part of $w\left(t, f_{n}\right)$ affecting the symbol, where $I_{[x, y]}(t)$ is the indicator function of the interval $[x, y] . \tilde{w}\left(t, f_{n}\right)$ admits the following Fourier series representation:

$$
\begin{aligned}
\tilde{w}\left(t, f_{n}\right) & =\sum_{k=-\infty}^{\infty} c_{k, n} e^{j 2 \pi k t / T} I_{[0, T]}(t) \\
c_{k, n} & =\frac{1}{T} \int_{0}^{T} \tilde{w}\left(t, f_{n}\right) e^{-j 2 \pi k t / T} d t
\end{aligned}
$$

where $\left\{c_{k, n}\right\}$ are random variables characterizing the effect of temporal variations in $\tilde{w}\left(t, f_{n}\right)$ over the symbol duration. $c_{k, n}$ represents the coupling coefficient between the transmitted signal on the $n$th subcarrier and the received signal corresponding to the subcarrier with frequency $f_{n}+k / T$. Substituting the Fourier series for $\tilde{w}\left(t, f_{n}\right)$ in (9) in the received signal (7), the received signal can be rewritten as

$r(t)=b \sum_{n=1}^{N} \sum_{k=-K_{l}}^{K_{u}} a_{n} c_{k, n} q(t) e^{j 2 \pi k t / T} e^{j 2 \pi f_{n} t}+n(t), \quad 0 \leq t \leq T$

where $K_{l}$ and $K_{u}$ are integers determined by the type of imperfection (discussed later) and $K_{l}+K_{u}+1$ is considered to be the number of strong coefficients that contribute to the Fourier expansion. Note that $K_{l}=K_{u}=0$ is an ideal system. In the presence of imperfections, more than one channel coefficients $\left\{c_{k, n}\right\}$ are associated with each subcarrier (see Fig. 2 for $\left.K_{l}=K_{u}=1\right)$. We also note that in general a relatively small number $\left(K_{u}+K_{l}+1\right)$ of subcarriers captures most of the energy in the dispersed subcarrier. The key idea of the paper is to restore the loss in performance and exploit diversity for each subcarrier by jointly processing adjacent subcarriers corresponding to significant $\left\{c_{k, n}\right\}$. This is equivalent to implementing a Rake receiver in the frequency domain as will be clarified in the sequel.

It can be readily shown that the general expression for $c_{k, n}$ in the presence of frequency offset, phase noise, and fast fading is

$$
c_{k, n}=\frac{1}{T} \int_{-B_{d}}^{B_{d}} H\left(\theta, f_{n}\right) G\left(\frac{k}{T}-f_{\text {off }}-\theta\right) d \theta
$$

where

$$
H\left(\theta, f_{n}\right)=\int \tilde{h}\left(t, f_{n}\right) e^{-j 2 \pi \theta t} d t
$$

and

$$
G(\theta)=\int_{0}^{T} e^{j \varrho(t)} e^{-j 2 \pi \theta t} d t
$$

We note that in this expansion model the basis functions are constant independent on imperfections. On the other hand, the channel coefficients depend on the type of imperfection.

\section{B. Degrees of Freedom in the Channel}

It may seem from the expression in (10) that the given model is over-parameterized since we have $N \times\left(K_{l}+K_{u}+1\right)$ expansion coefficients (i.e., $\left\{c_{k, n}\right\}$ ) to characterize the received signal of $N$ dimension. However, we note that the channel possesses only $L M\left(M=K_{l}+K_{u}+1\right)$ degrees of freedom. These degrees of freedom are dictated by the channel time and frequency selectivity. This implies that we only need to estimate $L M$ channel coefficients to capture the temporal and spectral variation in the channel in any given symbol duration. Since in most practical scenarios $L M \ll N$ (this will be clear in Sections V-VIII), the model is actually under-parameterized. It remains to explicitly show that the channel coefficients in (11) are derived from only $L M$ independent coefficients. We note that the received signal has an essentially bandwidth $B$. Hence, the part of $w(t, f)$ in (8) affecting the symbol is $\tilde{w}(t, f) I_{[-B / 2, B / 2]}$ and admits the following Fourier expansion [12]:

$$
\tilde{w}(t, f)=\sum_{l=0}^{L-1} w_{l}(t) e^{-j 2 \pi l f / B}, \quad-\frac{B}{2} \leq f \leq \frac{B}{2}
$$

where

$$
w_{l}(t)=\frac{1}{B} \int_{-B / 2}^{B / 2} \tilde{w}(t, f) e^{j 2 \pi l f / B} d f .
$$

From (14) and (15) in (9), $c_{k, n}$ can be rewritten as

$$
\begin{aligned}
c_{k, n} & =\sum_{l=0}^{L-1}\left(\frac{1}{T} \int_{0}^{T} w_{l}(t) e^{-j 2 \pi k t / T} d t\right) e^{-j 2 \pi l n / N} \\
& =\sum_{l=0}^{L-1} w_{k, l} e^{-j 2 \pi l n / N} .
\end{aligned}
$$

That is, by estimating $\left\{w_{k, l}\right\}, k=-K_{l}, \ldots, K_{u}, l=0, \ldots$, $L-1,\left\{c_{k, n}\right\}$ can be fully determined. This clearly shows that the received signal lies in a subspace of dimension $L M \ll N$. This fact will be used in Section IV to improve the channel estimates by a factor of $N / L M$. 
As seen from the representation in (10), the constants $K_{l}$ and $K_{u}$ determine the number of degrees of freedom as a result of time selectivity. Accurate values for $K_{l}$ and $K_{u}$ that capture the transmitted energy are useful in the context of noisy channel estimation. This is akin to finger management in Rake receivers [13]. Considering Rake fingers outside the $\left[-K_{l}, K_{u}\right]$ range will be mainly collecting noise and interference. This calls for an accurate determination of $K_{l}$ and $K_{u}$. We also note that values of $K_{l}$ and $K_{u}$ are dependent on the type of imperfection. For instance, we would expect frequency offset to require fewer channel coefficients as compared to fast fading or phase noise.

The values of $K_{l}$ and $K_{u}$ are determined based on the energy captured by the channel coefficients. The energy is dictated by the second-order statistics of the coefficients $\left\{c_{k, n}\right\}$ which are captured by the correlation function $\rho(k, n ; m, q)=E\left[c_{k, n} c_{m, q}^{*}\right]$ and vary with the type of imperfection. Since $H\left(\theta, f_{n}\right)$ and $G(\theta)$ in (11) are statistically independent, and $H\left(\theta, f_{n}\right)$ is uncorrelated for different values of $\theta$ [13], $\rho(k, n ; m, q)$ is shown in (17), at the bottom of the page, where

$$
\begin{aligned}
E\left[H\left(\theta, f_{n}\right) H^{*}\left(\theta^{\prime}, f_{q}\right)\right] & =\psi\left(\theta, f_{n}-f_{q}\right) \delta\left(\theta-\theta^{\prime}\right) \\
\psi(\theta, \Delta f) & =F T_{\{\Delta t\}}\{\phi(\Delta t, \Delta f)\} \\
& =\int \phi(\Delta t, \Delta f) e^{-j 2 \pi \theta \Delta t} d \Delta t \\
& =\Phi_{\theta}(\theta) \psi_{\Delta f}(\Delta f)
\end{aligned}
$$

and

$$
\varphi\left(\theta_{1}, \theta_{2}\right)=E\left[G\left(\frac{\theta_{1}}{T}\right) G^{*}\left(\frac{\theta_{2}}{T}\right)\right] .
$$

$\varphi\left(\theta_{1}, \theta_{2}\right)$ depends on phase noise statistics and is discussed in more detail in Section VII. The values of $K_{l}$ and $K_{u}$ for different combination of imperfections are obtained in Sections V-VII using explicit expressions of the correlation function defined in (17).

\section{Receiver Performance}

In this section, we analyze the performance of a coherent receiver employing maximal ratio combining (MRC) based on the proposed signal model in (10). Define the test statistics to be the projection of the received signal on the Fourier basis functions. The $n$th test statistic is

$$
\begin{aligned}
z_{n} & =\frac{1}{\sqrt{T}} \int_{0}^{T} r(t) e^{-j 2 \pi(n / T) t} d t \\
& =b a_{n} c_{0, n}+b \sum_{k=-K_{l}, k \neq 0}^{K_{u}} c_{k, n-k} a_{n-k}+v_{n}
\end{aligned}
$$

where $\left\{v_{n}\right\}$ are uncorrelated Gaussian random variables with zero mean and variance $\sigma^{2}$. We analyze two different receiver structures in the context of the proposed expansion model.

R1: This is the conventional receiver that ignores the effect of imperfections. It employs bit detection based only on the first term in the right-hand side of (20). Note that the first term is the desired signal component while the second term is the ICI due to imperfections.

R2: This is the MF receiver structure that in detecting the information on a given subcarrier, it jointly processes a subset of adjacent subcarriers.

Notice that in absence of imperfections (i.e., $K_{l}=K_{u}=0$ ), $z_{n}=b a_{n} c_{0, n}+v_{n}$. The receiver structure $\mathbf{R} \mathbf{1}$ is optimal in this case. It is convenient to express the test statistics (20) in matrix form ${ }^{2}$

$$
z_{n}=b\left(a_{n} c_{0, n}+\mathbf{a}_{n}^{T} \mathbf{c}_{n}\right)+v_{n}=b\left(g_{o}(n)+\grave{g}(n)\right)+v_{n}
$$

where



and

$\mathbf{c}_{n}=\left[\begin{array}{llllll}c_{-K_{u}, n+K_{u}}, & \ldots, & c_{-1, n+1} & c_{1, n-1}, & \ldots & c_{K_{l}, n-K_{l}}\end{array}\right]^{T}$.

Note that in the above definitions, $a_{i}=0$ if $i<1$ or $i>N$. Stacking the test statistics in one vector, we have

$$
\mathbf{z}=b \mathbf{g}+\mathbf{v}=b\left(\mathbf{g}_{o}+\grave{g}\right)+\mathbf{v}
$$

where $\mathrm{g}=\mathrm{g}_{o}+\mathrm{g}$ is the noise-free test statistics vector. $\mathrm{g}_{o}=$ $\mathbf{A}_{o} \mathbf{c}_{o}$ denotes the part of $\mathbf{g}$ corresponding to $k=0$ coefficients, that is, the desired signal in absence of imperfections. $\mathrm{g}=\mathbf{A c}$ corresponds to $k \neq 0$ and represents the ICI term within the test statistics. In these definitions

$$
\begin{aligned}
& \mathbf{A}_{o}=\operatorname{diag}\left(a_{1}, a_{2}, \ldots, a_{N}\right) \\
& \mathbf{c}_{o}=\left[\begin{array}{llll}
c_{0,1}, & c_{0,2}, & \cdots & c_{0, N}
\end{array}\right]^{T} \\
& \mathbf{A}=\left[\begin{array}{llll}
\mathbf{a}_{1}^{T} & \mathbf{0} & \cdots & \mathbf{0} \\
\mathbf{0} & \mathbf{a}_{2}^{T} & \cdots & \mathbf{0} \\
\vdots & \ddots & \ddots & \vdots \\
\mathbf{0} & \cdots & \mathbf{0} & \mathbf{a}_{N}^{T}
\end{array}\right]
\end{aligned}
$$

and

$$
\mathbf{c}=\left[\begin{array}{llll}
\mathbf{c}_{1}^{T}, & \mathbf{c}_{2}^{T}, & \cdots & \mathbf{c}_{N}^{T}
\end{array}\right]^{T} .
$$

Clearly, both $\mathrm{g}_{o}$ and g̀ carry information about the transmitted information bit. The MF receiver considers both terms in bit

${ }^{2} \mathbf{X}^{T}$ denotes the transpose and $\mathbf{X}^{H}$ the complex conjugate transpose of vector $\mathbf{x}$.

$$
\begin{aligned}
\rho(k, n ; m, q)= & E\left[c_{k, n} c_{m, q}^{*}\right]=\frac{1}{T^{2}} \int_{-B_{d}}^{B_{d}} \int_{-B_{d}}^{B_{d}} E\left[H\left(\theta, f_{n}\right) H^{*}\left(\theta^{\prime}, f_{q}\right)\right] \\
& \times E\left[G\left(\frac{k}{T}-f_{\text {off }}-\theta\right) G^{*}\left(\frac{m}{T}-f_{\text {off }}-\theta^{\prime}\right)\right] d \theta d \theta^{\prime} \\
= & \frac{1}{T^{2}} \int_{-B_{d}}^{B_{d}} \psi\left(\theta, f_{n}-f_{q}\right) \varphi\left(k-\theta T-f_{\text {off }} T, m-\theta T-f_{\text {off }} T\right) d \theta
\end{aligned}
$$


decision and is optimal. ${ }^{3}$ The bit decision based on MRC is given by

$$
\begin{aligned}
& \hat{b}=\operatorname{sign}\left\{\operatorname{real}\left[\mathbf{f}^{H} \mathbf{z}\right]\right\} \\
& \mathbf{f}=\left\{\begin{array}{ll}
\mathbf{g}_{o}, & \mathbf{R} \mathbf{1} \\
\mathbf{g}, & \mathbf{R 2}
\end{array}\right\} .
\end{aligned}
$$

If $\mathbf{f}$ is known at the receiver, the conditional probability of error $\left(P_{e}(\mathbf{f})\right)$ can be obtained as

$$
P_{e}(\mathbf{f})=Q\left(\sqrt{2 \frac{\mathbf{f}^{H} \mathbf{f}}{\sigma^{2}}}\right)
$$

where $Q(x)=(1 / \sqrt{2 \pi}) \int_{x}^{\infty} e^{-x^{2} / 2} d x$. For R1 in (27), the second term on the right-hand side of (20) is taken to be zero, i.e., the ICI term is not considered. This provides an upper bound on $\mathbf{R} 1$ performance. ${ }^{4}$

The unconditional $P_{e}$ is obtained by averaging the expression in (27) over the distribution of $\mathbf{f}$. If $\mathbf{f}$ is a complex Gaussian vector, $P_{e}$ has a closed form expression given by [13]

$$
P_{e}=\sum_{l=1}^{D} \frac{\pi_{l}}{2}\left[1-\sqrt{\frac{\mu_{l}}{1+\mu_{l}}}\right], \pi_{l}=\prod_{i=1, i \neq l}^{D} \frac{\mu_{l}}{\mu_{l}-\mu_{i}}
$$

where $\mu_{l}, l=1,2, \ldots, D$ are the nonzero eigenvalues of ${ }^{5}$ $\left(1 / \sigma^{2}\right) \mathbf{R}_{\mathbf{f}, \mathbf{f}}=\left(1 / \sigma^{2}\right) E\left[\mathrm{ff}^{H}\right]$. Using (26), these eigenvalues correspond to the nonzero eigenvalues of $\mathbf{R}_{\mathbf{g}_{o}, \mathbf{g}_{o}}$ for $\mathbf{R} \mathbf{1}$, and $\mathbf{R}_{\mathbf{g}, \mathbf{g}}$ for $\mathbf{R} 2$. In this case, the number of nonzero eigenvalues reflects the diversity order and is dependent on the second-order statistics of the coefficients $\mathbf{f}$ or equivalently the second-order statistics of $\left\{c_{k, n}\right\}$ that are captured by the correlation function in (17). If on the other hand $\mathbf{f}$ is not complex Gaussian (as in the case of phase noise), $P_{e}$ depends on higher order statistics and the closed form expression is not easy to obtain. For such cases, we resort to simulations to assess the receivers performance.

In the next section, we discuss in detail the problem of estimating the $\left\{c_{k, n}\right\}$ coefficients.

\section{Channel Estimation Issues}

In Section III-C, we showed the probability of error if $\mathbf{f}$ is perfectly estimated. In this section, we discuss the channel estimation requirements to implement $\mathbf{R} \mathbf{2}$. We adopt a pilot-aided channel estimation approach based on the least square (LS) criterion. We assume that the pilot test statistics suffer no interference from data. This can be made possible by having the pilot and data signals simultaneously transmitted on different spreading sequences with minimal cross correlation [18].

Using (16) in (20), the $n$th test statistic can be written as

$z_{n}=b \sum_{k=-K_{l}}^{K_{u}} \sum_{l=0}^{L-1} a_{n-k} w_{k, l} e^{-j 2 \pi l(n-k) / N}+v_{n}=\mathbf{q}_{n}^{T} \mathbf{w}+v_{n}$

where $\mathbf{q}_{n}=\left[\mathbf{q}_{n, 0}^{T}, \ldots, \mathbf{q}_{n, L-1}^{T}\right]^{T}, \quad \mathbf{q}_{n, l}=$ $\left[e^{-j 2 \pi l\left(n+K_{l}\right) / N} a_{n+K_{l}}, \ldots, e^{-j 2 \pi l\left(n-K_{u}\right) / N} a_{n-K_{u}}\right]^{T}, \mathbf{w}=$ $\left[\mathbf{w}_{0}^{T}, \ldots, \mathbf{w}_{L-1}^{T}\right]^{T}$, and $\mathbf{w}_{l}=\left[w_{-K_{l}, l}, \ldots, w_{K_{u}, l}\right]^{T}$. In the pilot phase, $b$ is known and without loss of generality

${ }^{3}$ The MF receiver is only optimal in the single-user scenario when one symbol is detected at a time (one shot detection).

${ }^{4}$ The ICI term in (20) will be included for $\mathbf{R} \mathbf{1}$ in the case of phase noise.

${ }^{5} \mathbf{R}_{\mathbf{x}, \mathbf{y}}=E\left[\mathbf{x y}^{H}\right]$. is chosen to be one. Cascading all test statistics in the pilot phase in one vector, we get

$$
\mathbf{z}_{\text {pilot }}=\mathrm{g}+\mathbf{v}=\mathrm{Qw}+\mathbf{v}
$$

where

$$
\mathbf{Q}=\left[\begin{array}{cccc}
\mathbf{q}_{1,0}^{T}, & \mathbf{q}_{1,1}^{T}, & \ldots, & \mathbf{q}_{1, L-1}^{T} \\
\mathbf{q}_{2,0}^{T}, & \ddots, & \ldots, & \mathbf{q}_{2, L-1}^{T} \\
\ldots, & \ddots, & \ddots, & \ldots \\
\mathbf{q}_{N, 0}^{T}, & \mathbf{q}_{N, 1}^{T}, & \ldots, & \mathbf{q}_{N, L-1}^{T}
\end{array}\right] .
$$

The LS channel estimate is, thus, given by

$$
\hat{\mathbf{w}}=\left(\mathbf{Q}^{H} \mathbf{Q}\right)^{-1} \mathbf{Q} \mathbf{z}_{\text {pilot }}
$$

and the estimated MF response is $\hat{\mathrm{g}}=\mathrm{Q} \hat{\mathrm{w}}$. The mean-square error (MSE) is defined as

$$
\begin{aligned}
\mathrm{MSE} & =E\left[\|\mathbf{g}-\hat{\mathbf{g}}\|^{2}\right] \\
& =\operatorname{trace}\left[\mathbf{Q}\left(\mathbf{Q}^{H} \mathbf{Q}\right)^{-1} \mathbf{Q}^{H}\right]=\operatorname{trace}\left[\mathbf{I}_{L M}\right]=L M \sigma^{2}
\end{aligned}
$$

where trace $[\mathbf{A}]$ is the sum of the diagonal entries in $\mathbf{A}$, and $\mathbf{I}_{P}$ is the $P \times P$ identity matrix. We note that if $\mathbf{g}$ is to be directly estimated from (30), the MSE would be $N \sigma^{2}$. That is utilizing the actual degrees of freedom in the channel improves the channel estimation error by a factor of $N / L M$.

Channel estimation can be enhanced by involving multiple symbols in the estimation process parallel to [18]. This is always possible within a window of adjacent symbols where the channel coefficients, across different symbols, are highly correlated. More details about this approach for channel estimation are outside the scope of this paper.

In subsequent sections, we explicitly determine the channel coefficients statistics for different types of imperfections that helps in determining $K_{l}$ and $K_{u}$.

\section{ReCEPTION IN THE PRESENCE OF FAST FADING}

When only fast fading is present, $w\left(t, f_{n}\right)=\tilde{h}\left(t, f_{n}\right), f_{\text {off }}=$ 0 , and $\varrho(t)=0$ in (8). The $\left\{c_{k, n}\right\}$ in (11) are given by [14], [12] $c_{k, n}=\int_{-B_{d}}^{B_{d}} H\left(\theta, f_{n}\right) \operatorname{sinc}\left(\left(\frac{k}{T}-\theta\right) T\right) e^{-j \pi T(k / T-\theta)} d \theta$.

The correlation function is given by

$\rho(k, n ; m, q)=$

$e^{-j \pi(k-m)} \int_{-B_{d}}^{B_{d}} \psi(\theta, \Delta f) \operatorname{sinc}\left(\left(\frac{k}{T}-\theta\right) T\right) \operatorname{sinc}\left(\left(\frac{m}{T}-\theta\right) T\right) d \theta$

where $\Delta f=f_{n}-f_{q}$ and $\psi(\theta, \Delta f)$ is defined in (18).

To determine $K_{l}$ and $K_{u}$, we study the power captured by the $c_{k, n}$ coefficient, that is $\rho(k, n ; k, n)$. It is clear that $\rho(k, n ; k, n)$ is symmetric in $k$. Thus, $K_{l}=K_{u}=K$. Fig. 3(a) shows the power in $\left\{c_{k, n}\right\}$, for any given $n$, and $B_{d} T=2$. It is evident that most of the energy (and diversity) is captured by $|k| \leq K=2$. The expression in (34) is a smoothing of $H\left(\theta, f_{n}\right)$ over $\theta$ with a sinc function having a null-to-null bandwidth $2 / T$. It follows that for any $n, K=\left\lceil B_{d} T\right\rceil$ is a good upper bound on the number of significant channel coefficients [14], [12]. For each 


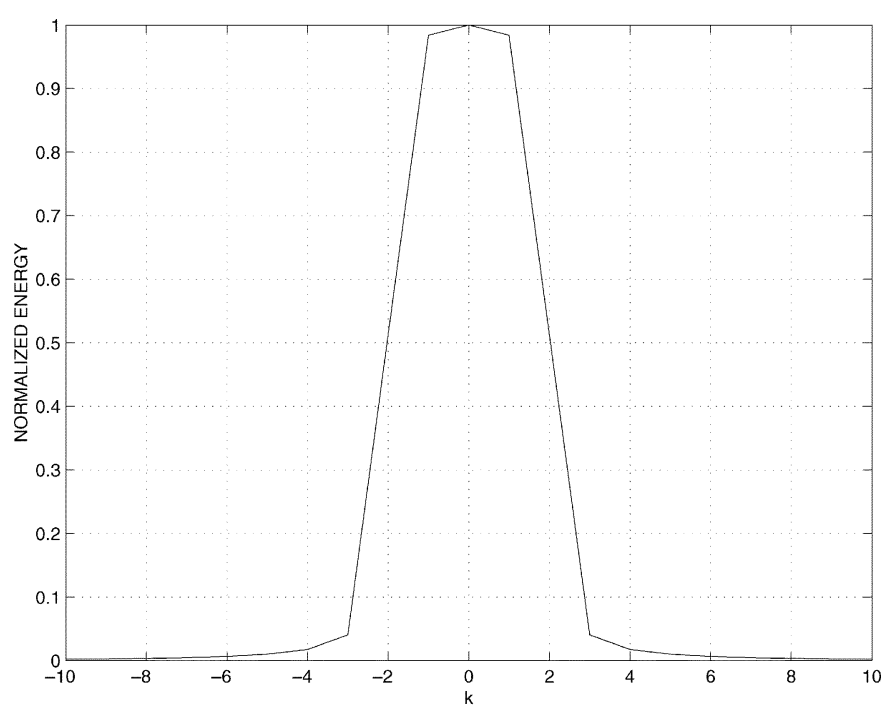

(a)

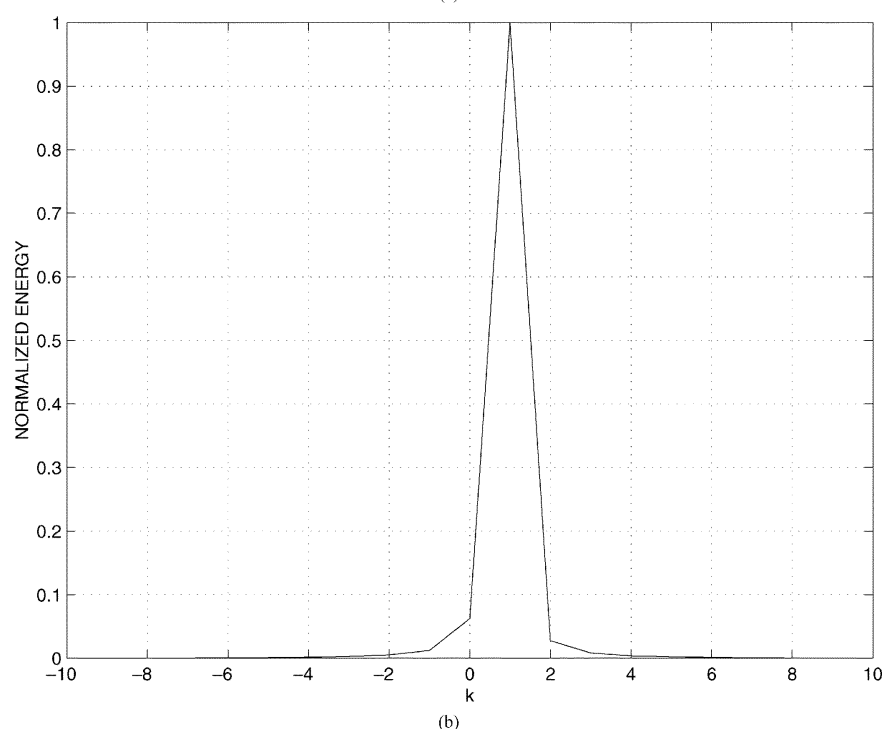

(b)

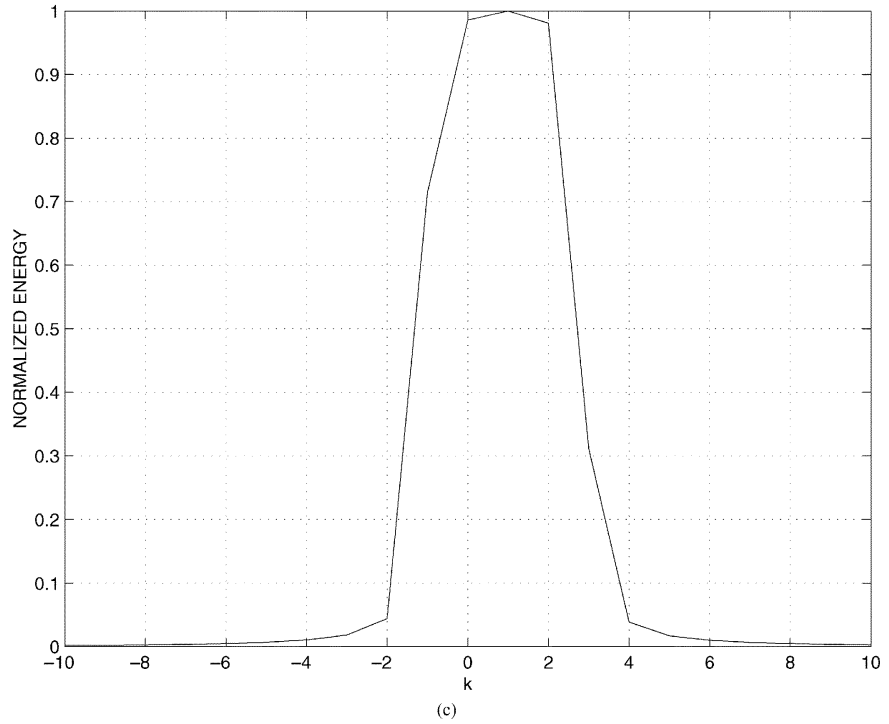

Fig. 3. Normalized energy captured by the $k$ th coefficient. (a) Fast fading channels with $B_{d} T=2$. (b) Slowly fading channel in the presence of frequency offset with $f_{\text {off }} T=0.8$. (c) $B_{d} T=2, f_{\text {off }} T=0.8$.

$n$, as long as $\left\{c_{k, n}, k=-K, \ldots, K\right\}$ are weakly correlated (which is the case of WSSUS channel), receivers $\mathbf{R} 2$ would exploit Doppler diversity due to the increase in the rank of $\mathbf{R}_{\mathbf{f}, \mathbf{f}}$ in $\mathbf{R 2}$ over that in $\mathbf{R} 1$. Thus, $\mathbf{R 2}$ provides better performance compared to $\mathbf{R} 1$ by collecting the dispersed energy as well as exploiting Doppler diversity [12].

\section{RECEPTION IN THE PRESENCE OF FREQUENCY OFFSET}

We now consider the case when there exists a frequency offset $f_{\text {off }}$ between the transmitter and receiver local oscillators (no phase noise). Subcarrier frequency offset causes severe attenuation in each subcarrier as well as ICI between the different subcarriers (see, e.g., [19]). We consider both slow and fast fading channels.

\section{A. Frequency Offset Correction in Slow Fading Channels}

In this case, $w\left(t, f_{n}\right)=\tilde{h}\left(f_{n}\right) e^{j 2 \pi f_{\text {off }} t}$ in (8) and the $\left\{c_{k, n}\right\}$ in (11) are given by

$$
c_{k, n}=\tilde{h}\left(f_{n}\right) \operatorname{sinc}\left(k-f_{\text {off }} T\right) e^{-j \pi\left(k-f_{\text {off }} T\right)} .
$$

From (36), the correlation function of the coefficients is given by

$$
\begin{aligned}
& \rho(k, n ; m, q)= \\
& \quad e^{-j \pi(k-m)} \psi_{\Delta f}(\Delta f) \operatorname{sinc}\left(k-f_{\text {off }} T\right) \operatorname{sinc}\left(m-f_{\text {off }} T\right)
\end{aligned}
$$

where $\Delta f=f_{n}-f_{q}$. Since most of the dominant coefficients in (36) lie in the main lobe of the sinc function, we have

$$
K_{l}=\left\lceil-f_{\text {off }} T\right\rceil \text { and } K_{u}=\left\lceil f_{\text {off }} T\right\rceil .
$$

Fig. 3(b) shows the energy captured by the $k$ th coefficient at $f_{\text {off }} T=0.8$. It is evident that (38) provides a good estimate for the range of dominant coefficients.

We note from (37) that in this case, the covariance matrix $\mathbf{R}_{\mathbf{f , f}}$ has the same rank for $\mathbf{R} \mathbf{1}$ and $\mathbf{R 2}$. This is because the statistics depend on $\psi_{\Delta f}(\Delta f)$ which does not depend on $f_{\text {off }}$. For any $n$ there is essentially a single dominant coefficient $c_{k, n}$-the frequency offset simply shifts the location of this coefficient. Hence, no additional diversity is available in this case. However, $\mathbf{R} 2$ restore the loss in performance encountered by $\mathbf{R} \mathbf{1}$ by collecting the dispersed energy.

\section{B. Frequency Offset Correction in Fast Fading Channels}

In this case, $w\left(t, f_{n}\right)=\tilde{h}\left(t, f_{n}\right) e^{j 2 \pi f_{\text {off }} t}$ in (8) and

$$
\begin{aligned}
& c_{k, n}= \\
& \quad \int_{-B_{d}}^{B_{d}} H\left(\theta, f_{n}\right) \operatorname{sinc}\left(\left(\frac{k}{T}-f_{\text {off }}-\theta\right) T\right) e^{-j \pi T\left(k / T-f_{\text {off }}-\theta\right)} d \theta .
\end{aligned}
$$

The correlation function of the coefficients is given by (40), shown at the bottom of the following page. Similar to Sections $\mathrm{V}$ and VI-A, $K_{l}=\left\lceil B_{d} T-f_{\text {off }} T\right\rceil$, and $K_{u}=\left\lceil B_{d} T+f_{\text {off }} T\right\rceil$. Fig. 3(c) show the distribution of energy over $k$ for $B_{d} T=2$ and $f_{\text {off }} T=0.8$. In this case, $\mathbf{R 2}$ not only restore the loss in performance due to frequency offset, but yield further improvement due to Doppler diversity. 


\section{ReCEPTION IN THE PRESENCE OF PhASe Noise}

We consider slow fading for simplicity in this case. As reported in many papers (see, e.g., [5]-[8]), the performance of OFDM systems degrades severely in the presence of phase noise and frequency offsets. Phase noise is particularly serious problem when relatively low-cost tuners are employed at the receiver.

In this case, the received signal for the first symbol can be expressed as

$$
\begin{aligned}
r(t) & =b \sum_{n=1}^{N} a_{n} \tilde{h}\left(f_{n}\right) q(t) e^{j 2 \pi f_{n} t} e^{j \varrho(t)}+n(t) \\
& =b x(t) e^{j \varrho(t)}+n(t)
\end{aligned}
$$

where $\varrho(t)$ is modeled as Brownian motion (or Wiener-Lévy) process with zero mean and variance $2 \pi \beta t$ [7], [20], [21]. If the phase noise realization is known at the receiver along with the channel coefficients $\left\{\tilde{h}\left(f_{n}\right)\right\}$, the optimal decision statistic is

$$
\zeta=\int_{0}^{T} x^{*}(t) e^{-j \varrho(t)} r(t) d t=b \int_{0}^{T}|x(t)|^{2} d t+\int_{0}^{T} x^{*}(t) n(t) d t
$$

which cancels phase noise and there is no loss in performance. In our framework, $w\left(t, f_{n}\right)=\tilde{h}\left(f_{n}\right) e^{j \varrho(t)}$ in (8) and the coefficients $\left\{c_{k, n}\right\}$ in the model (11) are given by

$$
c_{k, n}=\frac{1}{T} \tilde{h}\left(f_{n}\right) G\left(\frac{k}{T}\right)=\frac{1}{T} \tilde{h}\left(f_{n}\right) \int_{0}^{T} e^{j \varrho(t)} e^{-j(2 \pi k t / T)} d t .
$$

The performance can be restored by processing the discrete frequencies in (43) as opposed to the continuous processing in (42).

The correlation function defined in (17) is given by

$$
\rho(k, n ; m, q)=\frac{1}{T^{2}} \psi_{\Delta f}(\Delta f) \varphi(k, m)
$$

where $\Delta f=f_{n}-f_{q}, \psi_{\Delta f}\left(f_{n}-f_{q}\right)$ is defined in Section II-A, and

$$
\begin{aligned}
\varphi(x, y) & =E\left[G\left(\frac{x}{T}\right) G^{*}\left(\frac{y}{T}\right)\right] \\
& =E\left[\int_{0}^{T} \int_{0}^{T} e^{j(\varrho(\hat{t})-\varrho(\dot{u}))} e^{-j 2 \pi(x \hat{t}-y \dot{u}) / T} d \dot{t} d u ́\right] .
\end{aligned}
$$

We note that, for a particular $t, u, \varrho(t)-\varrho(u)$ is a Gaussian random variable with zero mean and variance $2 \pi \beta|t-u|$. It can be shown that

$$
E\left[e^{j(\varrho(t)-\varrho(u))}\right]=e^{-\pi \beta|t-u|}
$$

that is, the characteristic function of $\varrho(t)-\varrho(u)$ evaluated at a particular point. After some algebraic manipulations, the expression in (45) can be written as (47), shown at the bottom of the page, where $\gamma=\beta T, \alpha=(\gamma+j 2 x)(\gamma+j 2 y)$, and $\delta=(\gamma-j 2 x)(\gamma-j 2 y)$.

We note that $\mathbf{f}$ in (26) is no longer Gaussian in this case. Hence, the system performance does not only depend on $\mathbf{R}_{\mathbf{f}, \mathbf{f}}$ but rather on higher order statistics as well. However, the correlation function in (47) can be used to determine the number of coefficients $\left\{c_{k, n}\right\}$ (i.e., $K_{l}$ and $K_{u}$ ) needed to capture the dispersed energy. The power in the coefficients is proportional to

$$
\begin{aligned}
\varphi(x, x)=\frac{2 T^{2}}{\pi}\left\{\frac{\gamma}{\gamma^{2}+4 x^{2}}-\frac{\left(\gamma^{2}-4 x^{2}\right)}{\pi\left(\gamma^{2}+4 x^{2}\right)^{2}}+\frac{e^{-\pi \gamma}}{\pi}\right. \\
\\
\left.\times\left[\frac{\left(\gamma^{2}-4 x^{2}\right) \cos (2 \pi \gamma)-4 \gamma x \sin (2 \pi \gamma)}{\left(\gamma^{2}+4 x^{2}\right)^{2}}\right]\right\} .
\end{aligned}
$$

Special case of (47) for integer variables $k, m$ is

$$
\varphi(k, m)=\frac{2 T^{2}}{\pi^{2}} \frac{\left(\gamma^{2}-4 k m\right)\left(e^{-\pi \gamma}-1\right)}{\left(\gamma^{2}+4 k^{2}\right)\left(\gamma^{2}+4 m^{2}\right)}
$$

and the corresponding power at $k=m$ is

$$
\varphi(k, k)=\frac{2 T^{2}}{\pi}\left[\frac{\gamma}{\left(\gamma^{2}+4 k^{2}\right)}-\frac{\left(\gamma^{2}-4 k^{2}\right)\left(1-e^{-\pi \gamma}\right)}{\pi\left(\gamma^{2}+4 k^{2}\right)^{2}}\right] .
$$

Fig. 4(a) shows the normalized energy captured by the coefficients on a symbol duration $T$ as a function of the coefficient index $k$. As evident, the energy of the coefficients is symmetric around $k=0$. Hence, $K_{l}=K_{u}=K$. The function $\varphi(k, k)$ in (50) does not have a zero for any finite $k$, but it is monotonically decreasing with $k$. One way to choose $K$ is so that only those $\left\{c_{k, n}\right\}$ are included whose normalized power is above some preset threshold $\epsilon>0$. Fig. 4(b) shows $K$ as a function of $\beta T$ for different threshold values.

As mentioned earlier, due to non-Gaussianity of phase noise, the expression of $P_{e}$ in (28) is no longer valid. In this case, we assess system performance via simulations.

$$
\rho(k, n ; m, q)=e^{-j \pi(k-m)} \int_{-B_{d}}^{B_{d}} \psi(\theta, \Delta f) \operatorname{sinc}\left(\left(\frac{k}{T}-f_{\text {off }}-\theta\right) T\right) \times \operatorname{sinc}\left(\left(\frac{m}{T}-f_{\text {off }}-\theta\right) T\right) d \theta
$$

$$
\varphi(x, y)=\frac{T^{2}}{\pi^{2}}\left[\frac{T^{2}}{\pi^{2}} \frac{\gamma\left(e^{j 2 \pi(y-x)}-1\right)}{j\left(\gamma^{2}+4 x^{2}\right)(y-x)}+\frac{\left(\alpha e^{-\pi(\gamma-j 2 y)}-\delta e^{j 2 \pi(y-x)}+\delta e^{-\pi(\gamma+j 2 x)}-\alpha\right)}{\left(\gamma^{2}+4 x^{2}\right)\left(\gamma^{2}+4 y^{2}\right)}\right]
$$




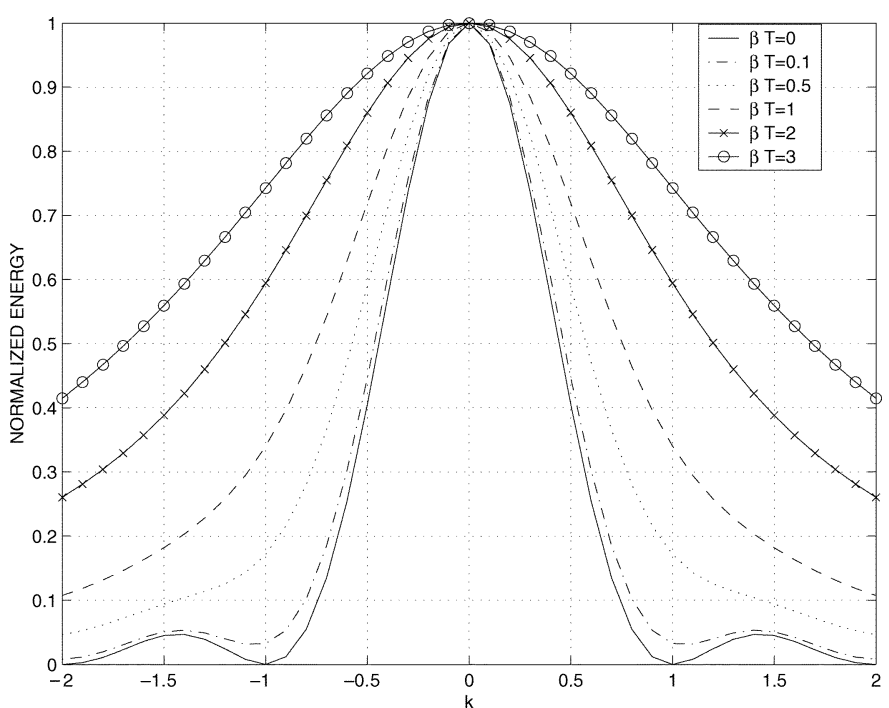

(a)

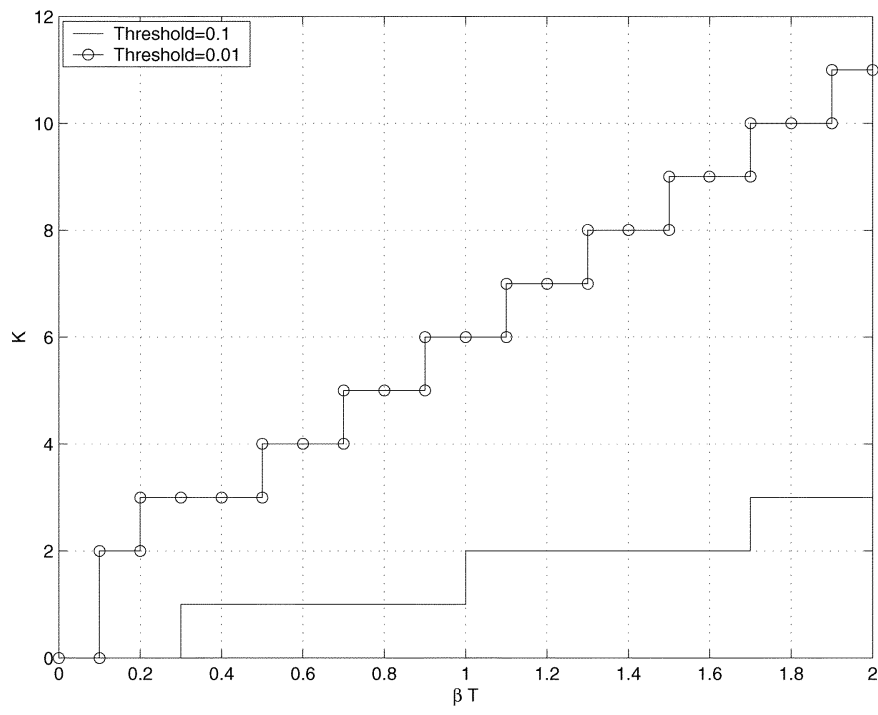

(b)

Fig. 4. (a) Normalized energy captured by the $k$ th coefficient in the presence of phase noise. (b) The number of coefficients $K$ whose normalized power exceeds a threshold $\epsilon=0.1,0.01$ as a function of $\beta T$.

\section{NUMERICAL RESULTS}

In this section, we address the performance of the proposed receiver via various examples emphasizing different types of imperfections. All the examples are based on a system using a Gold code with length $N=127$ that is used to modulate the different subcarriers. We consider a WSSUS channel model where the power in the different paths decays exponentially and the Doppler spectrum is flat for $\theta \in\left[-B_{d}, B_{d}\right]$ and zero elsewhere. The separability assumption in (2) is used in Examples 1 and 3 . All the results correspond to BPSK signaling. System performance is compared in terms of $P_{e}$ as a function of the parameters, $B_{d} T, f_{\text {off }} T$, and $\beta T$. In Examples 1, 2, 3, and 4, we compare the performance of the MF receiver $\mathbf{R} 2$ relative to conventional receiver $\mathbf{R} \mathbf{1}$ assuming perfect knowledge of $\mathbf{f}$ [defined in (26)]. In Example 5, we address the system performance of $\mathbf{R 2}$ (for phase noise imperfection) in the presence of channel estimation. In Examples 1, 2, and 3 (based on analytical results), the
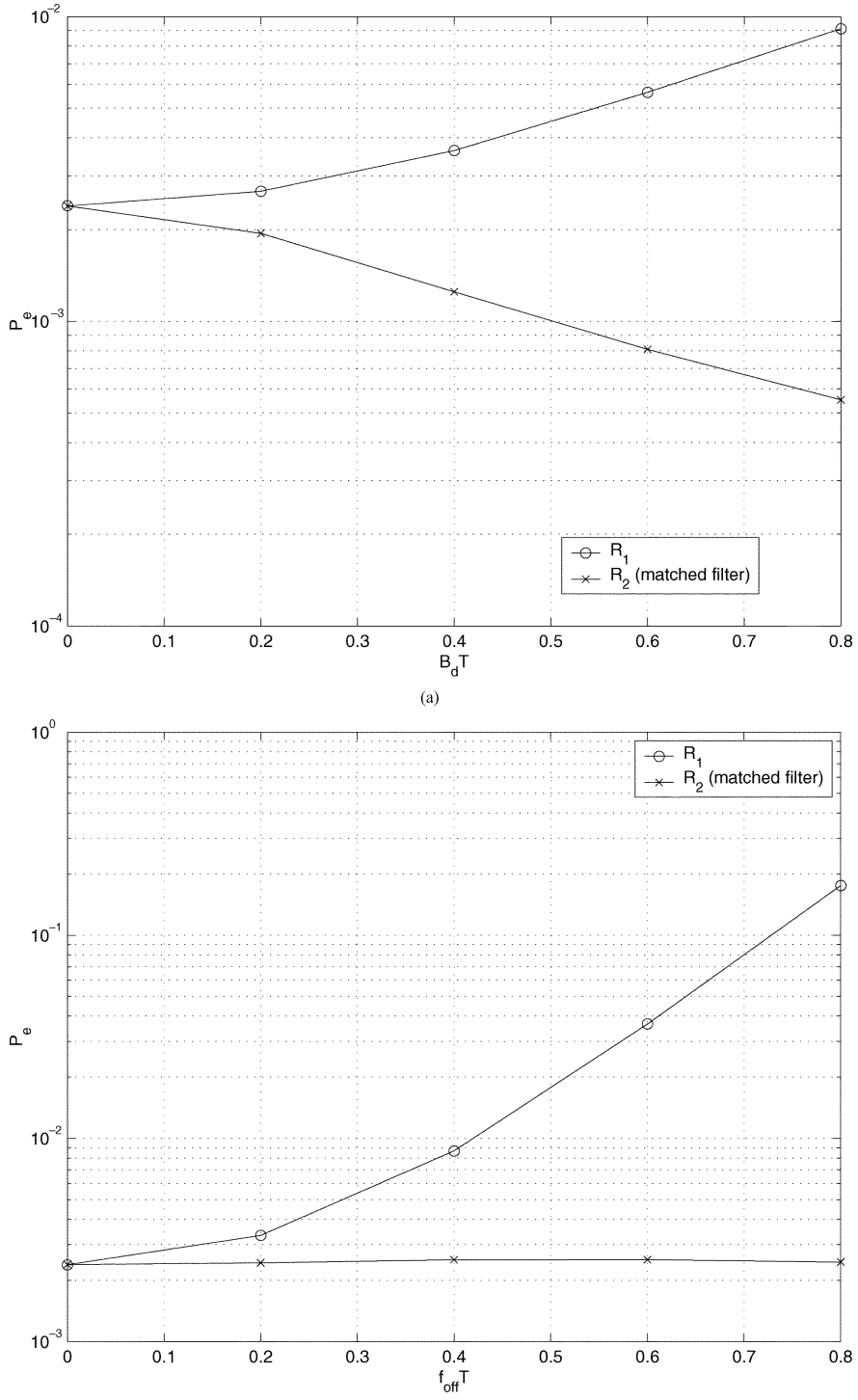

(b)

Fig. 5. (a) System performance as a function of fast fading parameter $B_{d} T$ at $\mathrm{SNR}=10 \mathrm{~dB}$. (b) System performance as a function of frequency offset parameter $f_{\text {off }} T$ at SNR $=10 \mathrm{~dB}$.

$P_{e}$ computation for $\mathbf{R} \mathbf{1}$ ignores ICI and is, thus, a lower bound on the $P_{e}$ of the conventional receiver operating in the presence of imperfections. In Examples 4 and 5, Monte Carlo simulations are used to assess system performance in the presence of phase noise. The phase noise is modeled as a random walk process with zero mean and variance $2 \pi \beta t$. In Example 4, the effects of ICI are included in $\mathbf{R} \mathbf{1}$ as well.

\section{A. Example 1}

We compare the two receivers in fast fading channels $\left(B_{d} T>\right.$ $0, f_{\text {off }} T=0$, and $\beta T=0$ ), SNR $=10 \mathrm{~dB}, L=6$, and $K=3$ which is sufficient to capture the signal energy for the range of $T B_{d}$ considered. Perfect knowledge of $\mathbf{f}$ automatically takes care of insignificant coefficient in the range $-K, K$. Fig. 5(a) shows the performance of the three receivers as a function of $B_{d} T$. The $P_{e}$ of the conventional receiver $\mathbf{R} 1$ increases monotonically with increasing $B_{d} T$. The performance of $\mathbf{R 2}$ improves with $B_{d} T$ due to Doppler diversity. 

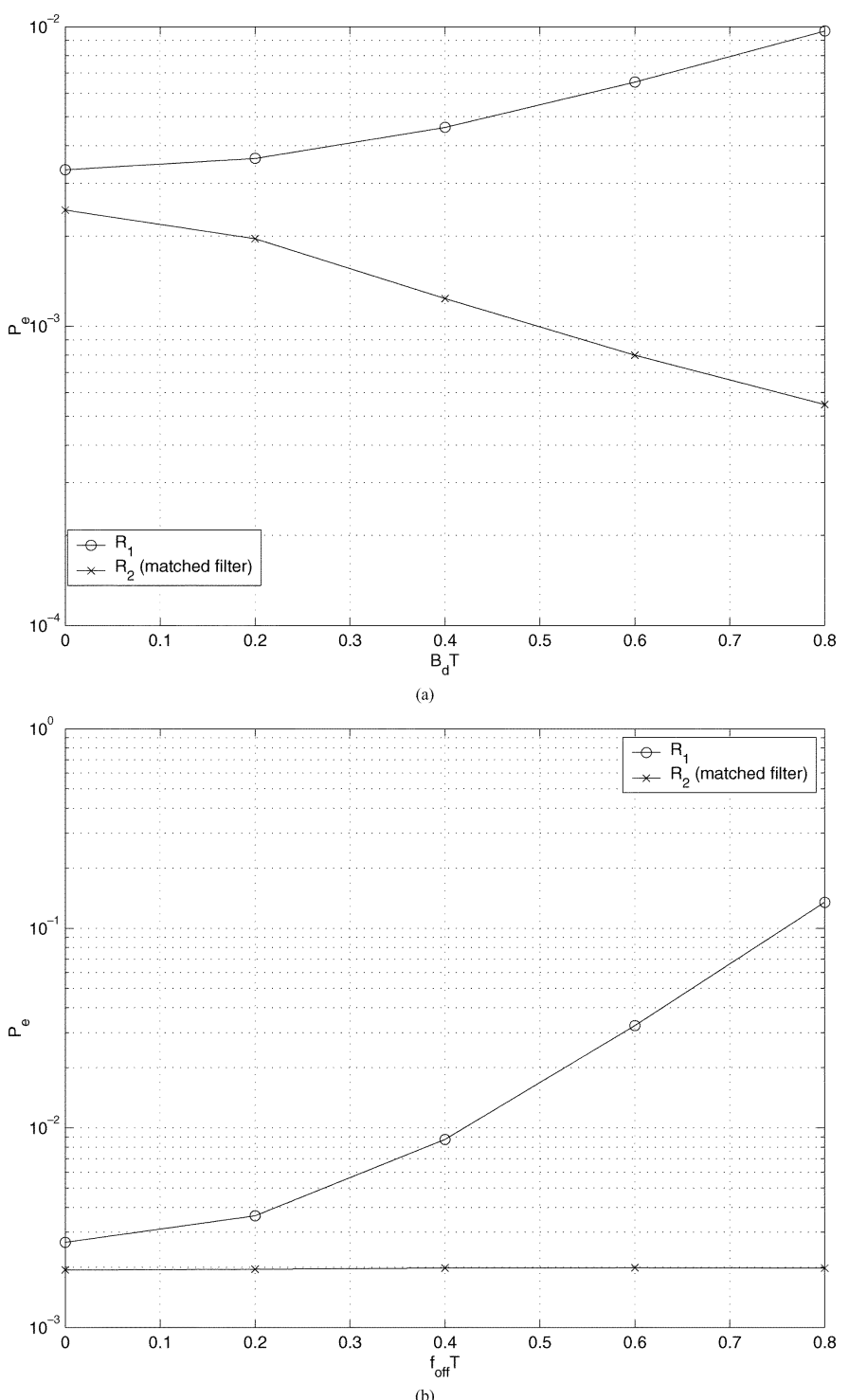

Fig. 6. (a) System performance as a function of $B_{d} T$ at SNR $=10 \mathrm{~dB}$ and $f_{\text {off }} T=0.2$. (b) System performance as a function of $f_{\text {off }} T$ at SNR $=10 \mathrm{~dB}$ and $B_{d} T=0.2$.

\section{B. Example 2}

This example compares the performance of the receivers in the presence of frequency offset in a slowly fading channel (i.e., $B_{d} T=0, f_{\text {off }} T>0$ and $\left.\beta T=0\right)$, SNR $=10 \mathrm{~dB}, L=$ 6 , and $K_{l}=K_{u}=3$. Fig. $5(\mathrm{~b})$ shows the performance as a function of $f_{\mathrm{off}} T$. The degradation in the performance of the conventional receiver R1 with increasing in $f_{\text {off }} T$ is evident. The performance of $\mathbf{R} \mathbf{2}$ is constant, unaffected by the increase of $f_{\text {off }} T$. It fully restores the performance to the offset-free case. However, as expected, no Doppler diversity is exploited in this case.

\section{Example 3}

This example considers effects of frequency offset in a fast fading channel (i.e., $B_{d} T>0, f_{\text {off }} T>0$ and $\beta T=0$ ), $\mathrm{SNR}=10 \mathrm{~dB}, L=6$, and $K_{l}=K_{u}=3$. Fig. 6(a) shows the performance as a function of $B_{d} T$ for fixed $f_{\text {off }} T=0.2$. Comparing the figure to Fig. 5(a), it is clear that the performance of
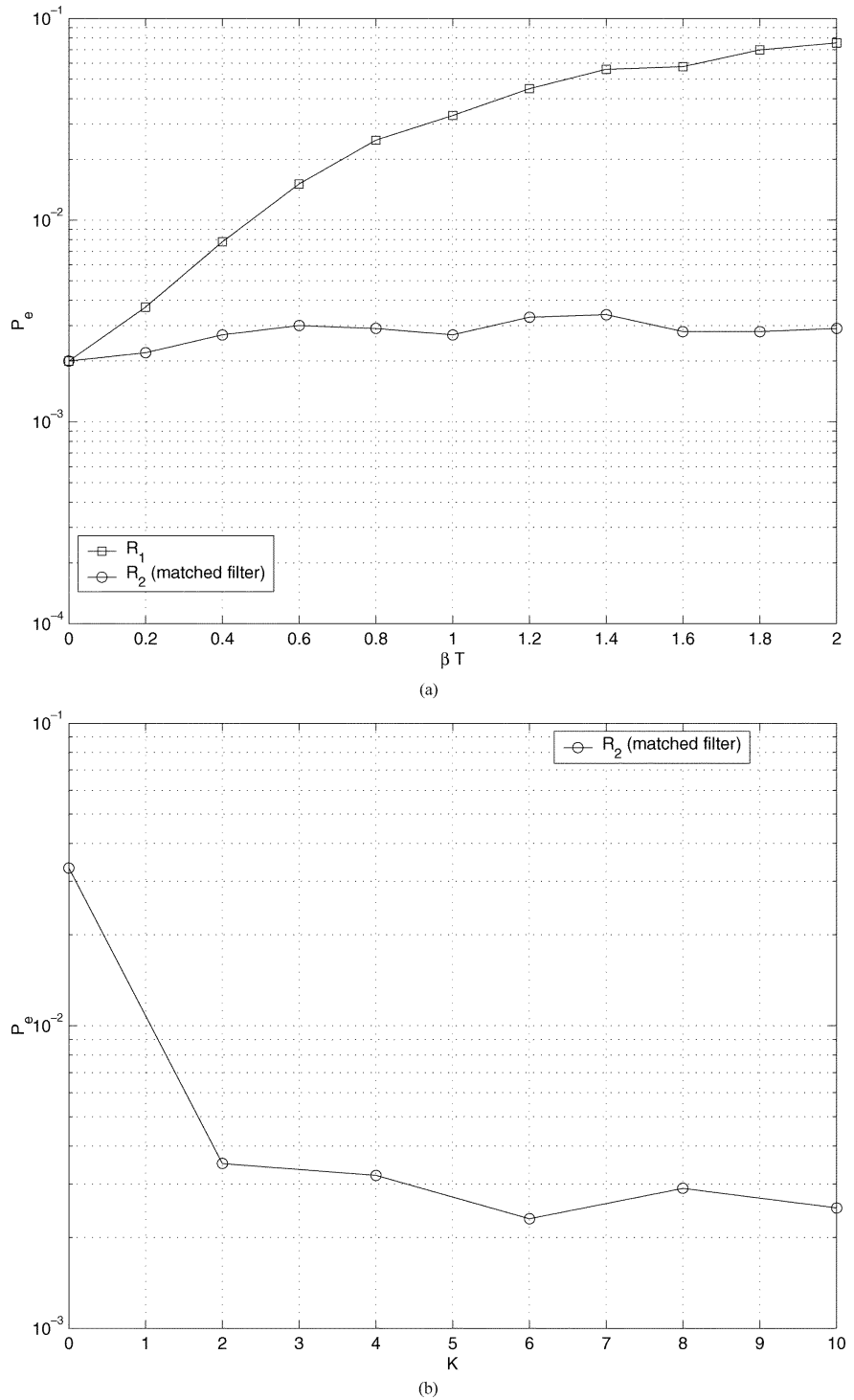

Fig. 7. Receiver performance in the presence of phase noise at SNR $=10 \mathrm{~dB}$ (a) $P_{e}$ as a function of $\beta T$ for $K=10$. (b) $P_{e}$ as a function of $K$ for $\beta T=0.8$.

$\mathbf{R 2}$ shows a similar trend with $\mathbf{R} 1$ showing a loss at $B_{d} T=0$ due to frequency offset. Fig. 6(b) shows the performance as a function of $f_{\text {off }} T$ at $B_{d} T=0.2$. Comparing with Fig. 5(b), it is clear that in addition to correcting for the frequency offset, $\mathbf{R 2}$ delivers improved performance due to Doppler diversity.

\section{Example 4}

This example compares the receivers in the presence of phase noise in a slowly fading channel (i.e., $B_{d} T=0, f_{\text {off }} T=0$ and $\beta T>0), \mathrm{SNR}=10 \mathrm{~dB}, L=6$, and $K=10$. The results are based on Monte Carlo simulations. Fig. 7(a) shows the system performance as a function of $\beta T$. The severe deterioration in performance of $\mathbf{R} \mathbf{1}$ is evident. The optimal performance of $\mathbf{R 2}$ is obvious since it is almost unaffected even at higher values of $\beta T$ because $\mathbf{R 2}$ collects all the dispersed energy ( $K=10$ is sufficiently large for the considered values of $\beta T$ ).

Fig. 7(b) assesses receiver performance as a function of $K$ at $\beta T=0.8$ for $\mathbf{R 2}$. We see that the performance almost saturates after $K=2$. This approximation corresponds to a threshold 




(a)

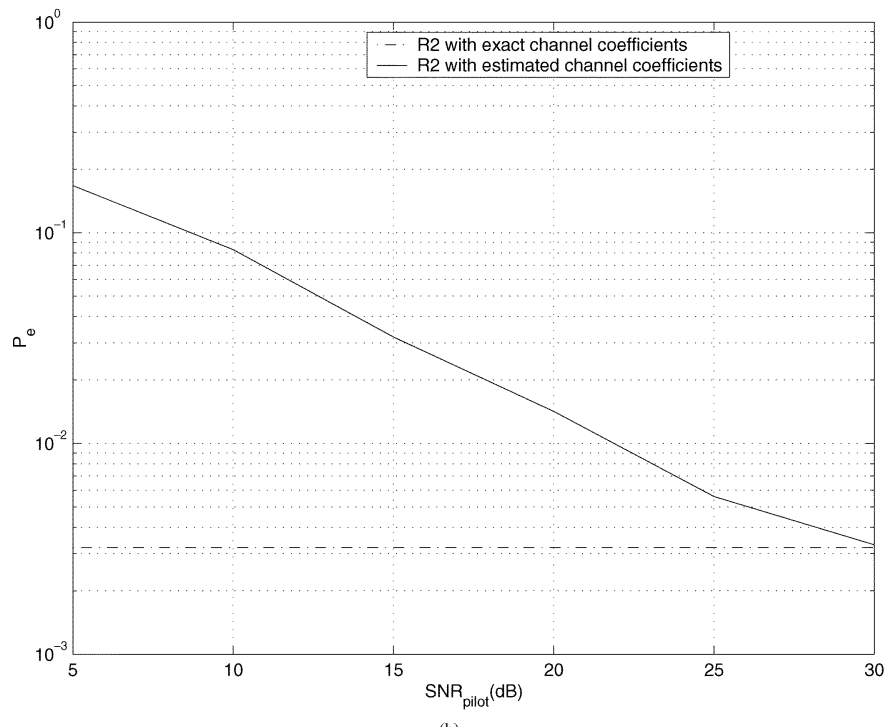

(b)

Fig. 8. Receiver performance with estimated channel coefficients at $\mathrm{SNR}_{\text {data }}=10 \mathrm{~dB}, K=3$, and $L=4$. (a) $P_{e}$ as a function of $\beta T$ for $\mathrm{SNR}_{\text {pilot }}=20 \mathrm{~dB}$. (b) $P_{e}$ as a function of $\mathrm{SNR}_{\text {pilot }}$ for $\beta T=0.5$.

$\epsilon=0.08$. This suggests that a relatively small subset of $K$ subcarriers in (43) is sufficient to restore the loss in performance due to moderate phase noise.

\section{E. Example 5}

This example shows the performance of $\mathbf{R} 2$ with ideal and estimated channel coefficients in the presence of phase noise. The examples considers a slowly fading channel (i.e., $B_{d} T=$ $0, f_{\text {off }} T=0$ and $\beta T>0$ ), the data signal-to-noise ratio (SNR) is taken to be $\mathrm{SNR}_{\text {data }}=10 \mathrm{~dB}, L=4$, and $K=3$. Channel estimation is done similar to Section IV where $L M=4 \times(3+$ $3+1)=28$ coefficients are required to be estimated. The results are based on Monte Carlo simulations.

Fig. 8(a) shows the $P_{e}$ versus $\beta T$ for $\mathrm{SNR}_{\text {pilot }}=20 \mathrm{~dB}$. Fig. 8(b) shows the $P_{e}$ versus $\mathrm{SNR}_{\text {pilot }}$ for $\beta T=0.5$. It is clear in Fig. 8(a) that $\mathbf{R 2}$, in the presence of channel estimation, still restores the performance loss due to phase noise imperfection. However, the figure shows a gap between the performance of exact and estimated channel coefficients. As mentioned before, this gap can be improved by building the channel estimates on multiple adjacent symbols. It also improves by increasing the pilot SNR, as shown in Fig. 8(b).

\section{CONCLUSION}

We have proposed an integrated receiver for MC-CDMA system operating in the presence of commonly encountered imperfections-frequency offsets, fast fading, and phase noise. The receiver structure is based on a canonical model for the received signal in terms of subcarrier basis functions that implements the MF, hence capturing the effects of all imperfections. In the case of frequency offset and phase noise, the proposed receiver fully restores the loss in performance of the conventional receivers. In the presence of fast fading, the proposed receiver actually delivers improved performance by exploiting Doppler diversity. The problem of channel estimation was discussed and a simple LS-based estimation scheme was provided. Our results demonstrate that significant gains in performance can be attained as long as accurate estimates of the channel coefficients are available. The universal structure of the proposed receiver eliminates the need to correct for each type of imperfection separately. We believe that the framework is extendable to covering other imperfections that causes ISI such as nonlinearities in power amplifiers.

\section{REFERENCES}

[1] S. Hara and R. Prasad, "Overview of multicarrier CDMA," IEEE Commun. Mag., vol. 35, pp. 126-133, Dec. 1997.

[2] N. Yee, J.-P. Linnartz, and G. Fettweis, "Multicarrier CDMA in indoor wireless radio networks," in Proc. IEEE PIMRC, Sept. 1993, pp. $109-113$.

[3] V. M. DaSilva and E. S. Sousa, "Performance of orthogonal CDMA codes for quasisynchronous communication systems," in IEEE ICUPC'93, Ottawa, Canada, Oct. 1993, pp. 995-999.

[4] L. Vandendorpe, "Multitone direct sequence CDMA for frequency selective fading channels," in 1st Symp. Communications Vehicular Technology, Delft, The Netherlands, Oct. 1993.

[5] T. Pollet, M. V. Bladel, and M. Moeneclaey, "BER senstivity of OFDM systems to carrier frequency offset and wiener phase noise," IEEE Trans. Commun., vol. 43, pp. 191-193, Feb./Mar./Apr. 1995.

[6] P. Robertson and S. Kaiser, "Analysis of the effect of phase-noise in orthogonal frequency division multiplex (OFDM) systems," in Proc. IEEE ICC '95, Seattle, WA, June 1995, pp. 1652-1657.

[7] L. Tomba and W. A. Krzymien, "Senstivity of the MC-CDMA access scheme to carrier phase noise and frequency offset," IEEE Trans. Veh. Technol., vol. 48, pp. 1657-1665, Sept. 1999.

[8] T. Pollet, M. Moeneclaey, I. Jeanclaude, and H. Sari, "Effect of carrier phase jitter on single-carrier and multi-carrier QAM systems," in Proc. ICC 1995, Seattle, WA, June 1995, pp. 1046-1050.

[9] C. Muschallik, "Influence of RF oscillators on an OFDM signal," IEEE Trans. Consumer Electron., vol. 41, pp. 592-603, Aug. 1995.

[10] P. Robertson and S. Kaiser, "Analysis of the loss of orthogonality through Doppler spread in OFDM systems," in Proc. GLOBECOM '99, Brazil, Dec. 1999, pp. 1-10.

[11] T. A. Kadous and A. M. Sayeed, "Equivalence of linear MMSE detection in DS-CDMA and MC-CDMA systems over time and frequency selective channels," EURASIP J. Appl. Signal Processing (Special Issue on Multiuser Detection and Blind Estimation), no. 12, pp. 1335-1354, Dec. 2002.

[12] A. M. Sayeed and B. Aazhang, "Joint multipath-Doppler diversity in mobile wireless communications," IEEE Trans. Commun., vol. 47, pp. 123-132, Jan. 1999.

[13] J. G. Proakis, Digitial Communications, 3rd ed. New York: McGrawHill, 1995.

[14] P. A. Bello, "Measurement of random time-variant linear channels," IEEE Trans. Inform. Theory, vol. IT-15, pp. 469-475, July 1969. 
[15] Y. Li, L. Cimini, and N. R. Sollenberger, "Robust channel estiamtion for OFDM systems with rapid dispersive fading channels," IEEE Trans. Commun., vol. 46, pp. 902-915, July 1998.

[16] H. Steendarn and M. Moeneclaey, "Analysis and optimization of the performance of OFDM on frequency-selective time-selective fading channels," IEEE Trans. Commun., vol. 47, pp. 1811-1819, Dec. 1999.

[17] G. Giannakis and C. TepedelenlioGlu, "Basis expansion models and diversity techniques for blind identification and equalization of timevarying channels," Proc. IEEE, vol. 86, pp. 1969-1985, Oct. 1998.

[18] M. Baissas and A. Sayeed, "Pilot-based estimation of time-varying multipath channels for coherent CDMA receivers," IEEE Trans. Signal Processing, vol. 50, pp. 2037-2049, Aug. 2002.

[19] J. Armstrong, "Analysis of new and existing methods of reducing intercarrier interference due to carrier frequency offset in OFDM," IEEE Trans. Commun., vol. 47, pp. 365-369, Mar. 1999.
[20] G. J. Foschini and G. Vannucci, "Characterizing filtered light waves corrupted by phase noise," IEEE Trans. Inform. Theory, vol. IT-34, pp. 1437-1448, Nov. 1988.

[21] A. Papoulis, Probability, Random Variables and Stochastic Processes, 3rd ed. New York: McGraw-Hill, 1991.

Tamer A. Kadous (S'95-M'01), photograph and biography not available at the time of publication.

Akbar M. Sayeed (S'96-M'97-SM'02), photograph and biography not available at the time of publication. 